\section{Drogennachweis mit Teststreifen}

C. Vidal ${ }^{1}$ und W.-R. Külpmann ${ }^{2}$

${ }^{1}$ Landeskriminalamt Niedersachsen, Dezernat 53 „Chemie“, Hannover, Deutschland

${ }^{2}$ Hannover, Deutschland

Englischer Begriff drug screening by test strips

Beschreibung Der Nachweis von Pharmaka im Urin mit Teststreifen ist scheinbar leicht durchführbar. Tatsächlich müssen zahlreiche Aspekte berücksichtigt werden, um falsch positive und falsch negative Befunde zu vermeiden bzw. zu erkennen.

Viele nachzuweisende Substanzen werden größtenteils metabolisiert und konjugiert, bevor sie renal eliminiert werden. Ein Hydrolyseschritt vor Analyse mittels Teststreifen ist jedoch nicht vorgesehen und die Kreuzreaktivität gegenüber Konjugaten meist schwach. Es resultiert ein deutlich höherer
Cut-off-Wert als der Anwender annimmt: Der angegebene Cut-off-Wert bezieht sich auf die freie (unkonjugierte) Substanz des Kalibrators.

Die Beurteilung erfolgt subjektiv visuell und beim Feldeinsatz häufig bei ungenügender Beleuchtung. Ein positiver Befund ist meist gekennzeichnet durch eine fehlende Farbbande, schwach (?) gefärbte Banden gelten als negativ.

Eine differenzierende Auswertung wie bei einem primär quantitativen Messsignal ist nicht möglich.

Eine Kontrolllinie auf dem einzelnen Teststreifen ist ein Indiz, dass der Analysenvorgang regelrecht abgelaufen ist. Eine regelmäßige Qualitätssicherung mit Kontrollurin wird dennoch von den Herstellern mit Recht für notwendig gehalten.

\section{Literatur} Külpmann WR (2003) Nachweis von Drogen und Medikamenten im
Urin mittels Schnelltests. Dtsch Ärztebl 100:A1138-A1140 